\title{
A GENERALIZATION OF MULTIVALENT FUNCTIONS WITH NEGATIVE COEFFICIENTS
}

\author{
M.K. AOUF \\ Faculty of Science \\ Department of Mathematics \\ University of Mansoura \\ Mansoura, Egypt \\ (Received January 6, 1987)
}

ABSTRACT. Let $T_{p}$ be the class of analytic and p-valent functions which can be expressed in the form

$$
f(z)=z^{p}-\sum_{n=1}^{\infty}\left|a_{n+p}\right| z^{n+p},|z|<1 .
$$

The subclasses $T_{p}^{*}(A, B, \alpha)$ and $C_{p}(A, B, \alpha)$ of $T_{p}$ have been considered. Sharp results concerning coefficient estimates, distortion and covering theorems are obtained. The radius of convexity for the class $T_{p}{ }^{*}(A, B, \alpha)$ is determined. It is further proved that the classes $T_{p}^{*}(A, B, \alpha)$ and $C_{p}(A, B, \alpha)$ are closed under arithmetic mean and convex linear combinations.

KEY WORDS AND PHRASES. P-valent, Analytic, Radius of Convexity.

1980 AMS SUBJECT CLASSIFICATION CODES. 30A32, $30 \mathrm{~A} 36$.

1. INTRODUCTION.

Let $s_{p}(p>1)$ denote the class of functions of the form

$f(z)=z^{p}+\sum_{n=1}^{\infty} a_{p+n} z^{p+n}$ which are analytic and p-valent in the unit disc

$U=\{z:|z|<1\}$. A function $f$ is said to be subordinate to a function $F(f<F)$ if there exists an analytic function $\phi(z)$ with $|\phi(z)|<|z|, z \varepsilon U$, such that $\mathrm{f}=\mathrm{f} \cdot \phi$.

For $A, B$ fixed, $-1<A<B<1$, and $0<\alpha<p$, we say that $f \in S_{p}^{*}(A, B, \alpha)$ if and only if

$$
\frac{z f^{\prime}(z)}{f(z)}<\frac{p+[p B+(A-B)(p-\alpha)] z}{1+B z}, z \varepsilon U,
$$


or equivalently $f \in S_{p}^{*}(A, B, \alpha)$ if and only if

$$
\left|\frac{\frac{z f^{\prime}(z)}{f(z)}-p}{B \frac{z f^{\prime}(z)}{f(z)}-[p B+(A-B)(p-\alpha)]}\right|<1, z \varepsilon U \text {. }
$$

Further $f$ is said to belong to the class $K_{p}(A, B, \alpha)$ if and only if $\frac{z f^{\prime}(z)}{p} \varepsilon S_{p}^{*}(A, B, \alpha)$.

Let $T_{p}$ denote the subclass of $S_{p}$ consisting of functions analytic and $p$-valent which can be expressed in the form

$$
\begin{gathered}
f(z)=z^{p}-\sum_{n=1}^{\infty}\left|a_{p+n}\right| z^{p+n} \text {, and we set } \\
T_{p}^{*}(A, B, \alpha)=S_{p}^{*}(A, B, \alpha) \quad T_{p} \text { and } C_{p}(A, B, \alpha)=\operatorname{Kp}(A, B, \alpha) T_{p} \cdot
\end{gathered}
$$

Silverman [3], Gupta and Jain [2] and Silverman and Silvia [4,5] have studied Certain subclasses of univalent functions with negative coefficients. Also Goel and Sohi [1] have studied certain subclasses of multivalent functions with negative coefficients. In this paper we obtain coefficient estimates, distortion and covering theorems for the classes $T_{p}^{*}(A, B, \alpha)$ and $C_{p}(A, B, \alpha)$. We also determine the radius of convexity for the class ${ }^{*}{ }_{p}^{*}(A, B, \alpha)$. It is further shown that the classes $T_{p}^{*}(A, B, \alpha)$ and $C_{p}(A, B, \alpha)$ are ${ }^{*}$ closed under arithmetic mean and convex linear combinations. By taking $\alpha=0$, we get results due to Goel and Sohi [1] and by assigning specific values to $A$ and $B$ and taking $p=1$, we get results due to Silverman [3] and Gupta and Jain [2].

2. COEFFICIENT INEQUALITIES.

THEOREM 1. A function $f(z)=z^{p}-\sum_{n=1}^{\infty}\left|a_{p+n}\right| z^{p+n}$ is in $T_{p}^{*}(A, B, \alpha)$ if and only if

$$
\sum_{n=1}^{\infty}[(1+B) n+(B-A)(p-\alpha)]\left|a_{p+n}\right|<(B-A)(p-\alpha) .
$$

The result is sharp.

PROOF. Let $|z|=1$, then

$$
\begin{aligned}
& \mid z f^{\prime}(z)-p f(z)|-| B z f^{\prime}(z)-[p B+(A-B)(p-\alpha)] f(z) \mid \\
&=\left|\sum_{n=1}^{\infty}-n\right| a_{p+n}\left|z^{p+n}\right|-\mid(B-A)(p-\alpha) z^{p} \\
&-\sum_{n=1}^{\infty}[n B+(B-A)(p-\alpha)]\left|a_{p+n}\right| z^{p+n} \mid \\
&<\sum_{n=1}^{\infty}[(1+B) n+(B-A)(p-\alpha)]\left|a_{p+n}\right|-(B-A)(p-\alpha)<0 .
\end{aligned}
$$

Hence by the principle of maximum modulus $f(z) \in T_{p}^{*}(A, B, \alpha)$.

Conversely, suppose that 


$$
\begin{aligned}
& \left|\frac{\frac{z f^{\prime}(z)}{f(z)}-p}{\text { B. } \frac{z f^{\prime}(z)}{f(z)}-[p B+(A-B)(p-\alpha)]}\right| \\
& =\left|\frac{\sum_{n=1}^{\infty} n\left|a_{p+n}\right| z^{p+n}}{(B-A)(p-\alpha) z^{p}-\sum_{n=1}^{\infty}[n B+(B-A)(p-\alpha)]\left|a_{p+n}\right| z^{p+n}}\right|<1, \quad \text { z } \varepsilon \text { U. }
\end{aligned}
$$

Since $|\operatorname{Re} z|<|z|$ for all $z$, we have

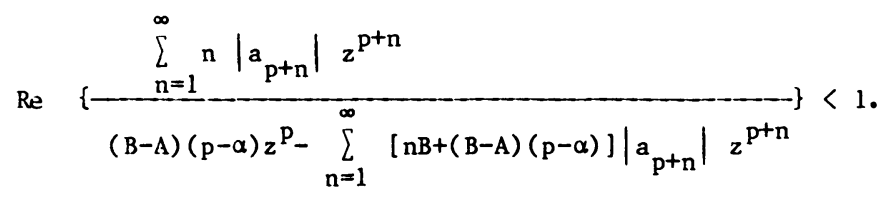

Choose values of $z$ on the real axis so that $\frac{z f^{\prime}(z)}{f(z)}$ is real. Upon clearing the denominator in (2.2) and letting $z+1$ through real values, we obtain

$$
\sum_{n=1}^{\infty} n\left|a_{p+n}\right| \leqslant\left\{(B-A)(p-\alpha)-\sum_{n=1}^{\infty}[n B+(B-A)(p-\alpha)]\left|a_{p+n}\right|\right\}
$$

which implies that

$$
\sum_{n=1}^{\infty}[(1+B) n+(B-A)(p-\alpha)]\left|a_{p+n}\right|<(B-A)(p-\alpha) .
$$

The function

$$
f(z)=z^{p}-\sum_{n=1}^{\infty} \frac{(B-A)(p-\alpha)}{(1+B) n+(B-A)(p-\alpha)} z^{p+n}
$$

is an extremal function.

COROLLARY 1. If $f \in T_{p}^{*}(A, B, \alpha)$ then $\left|a_{p+n}\right|<\frac{(B-A)(p-\alpha)}{(1+B) n+(B-A)(p-\alpha)}$, with

equality only for functions of the form $f(z)=z^{p}-\frac{(B-A)(p-\alpha)}{(1+B) n+(B-A)(p-\alpha)} z^{p+n}$. if

COROLlaRY 2. A function $f(z)=z^{p}-\sum_{n=1}^{\infty}\left|a_{p+n}\right| z^{p+n}$ is in $C_{p}(A, B, \alpha)$ if and only

$$
\sum_{n=1}^{\infty}\left(\frac{n+p}{p}\right)[(1+B) n+(B-A)(p-\alpha)]\left|a_{p+n}\right|<(B-A)(p-\alpha) .
$$

PROOF. It is well known that $f \in C_{p}(A, B, \alpha)$ if and only if $\frac{z f^{\prime}(z)}{p} \varepsilon T_{p}^{*}(A, B, \alpha)$. Since $\quad \frac{z f^{-}(z)}{p}=z^{p}-\sum_{n=1}^{\infty}\left(\frac{n+p}{p}\right)\left|a_{p+n}\right| z^{p+n}$

we may replace $\left|a_{p+n}\right|$ with $\left(\frac{n+p}{p}\right)\left|a_{p+n}\right|$ in Theorem 1. 
3. REPRESENTATION FORMULA.

THEOREM 2. A function $f(z)=z^{p}-\sum_{n=1}^{\infty}\left|a_{n+p}\right| z^{n+p}$ is in $T_{p}^{*}(A, B, \alpha)$ if and only if

$$
f(z)=z^{p} \exp \left\{(B-A)(p-\alpha) \int_{0}^{z} \frac{\phi(t)}{1-B t \phi(t)} d t\right\} \text {, }
$$

where $\phi(z)$ is analytic in $U$ and satisfies $|\phi(z)|<1, z \varepsilon U$.

PROOF. Let $f(z) \varepsilon T_{p}^{*}(A, B, \alpha)$, then

$$
\left|\frac{z f^{\prime}(z)}{\mid \frac{f(z)}{B(z)}-p}\right|<1, z \in U \text {. }
$$

Since the absolute value vanishes for $z=0$, we have

$$
\left|\frac{z f^{\prime}(z)}{\mid \frac{f(z)}{z .} p}\right|=h(z)
$$

where $h(z)$ is analytic in $U$ and $|h(z)|<1$ for $z \in U$. Integrating (3.2) with $h(z)=z \phi(z)$ we find that

$$
f(z)=z^{p} \cdot \exp \left\{(B-A)(p-\alpha) \int_{0}^{z} \frac{\phi(t)}{1-B t \phi(t)} d t\right\}
$$

The converse is obtained by differentiating (3.1).

4. Distortion AND COVERING THEOREMS FOR $T_{p}^{*}(A, B, \alpha)$ and $C_{p}(A, B, \alpha)$.

THEOREM 3. If $f(z) \varepsilon T_{p}^{*}(A, B, \alpha)$, then

$$
\begin{aligned}
& r^{p}-\frac{(B-A)(p-\alpha)}{1+B+(B-A)(p-\alpha)} r^{p+1}<|f(z)|< \\
& r^{p}+\frac{(B-A)(p-\alpha)}{1+B+(B-A)(p-\alpha)} r^{p+1} \quad(|z|=r),
\end{aligned}
$$

with equality for $f(z)=z^{p}-\frac{(B-A)(p-\alpha)}{1+B+(B-A)(p-\alpha)} z^{p+1}(z= \pm r)$.

PROOF. From Theorem 1, we have

$$
[1+B+(B-A)(p-\alpha)] \sum_{n=1}^{\infty}\left|a_{p+n}\right|<\sum_{n=1}^{\infty}[(1+B) n+(B-A)(p-\alpha)]\left|a_{p+n}\right|<(B-A)(p-\alpha) .
$$

This implies that

$$
\sum_{n=1}^{\infty}\left|a_{p+n}\right|<\frac{(B-A)(p-\alpha)}{1+B+(B-A)(p-\alpha)}
$$

Thus

$$
\begin{aligned}
|f(z)| & <|z|^{p}+\sum_{n=1}^{\infty}\left|a_{p+n}\right||z|^{p+n} \\
& <r^{p}\left(1+r \sum_{n=1}^{\infty}\left|a_{p+n}\right|\right)
\end{aligned}
$$




$$
<r^{p}+\frac{(B-A)(p-\alpha)}{1+B+(B-A)(p-\alpha)} r^{p+1}
$$

Similarly,

$$
\begin{aligned}
|f(z)| & >|z|^{p}-\sum_{n=1}^{\infty}\left|a_{p+n}\right| \cdot|z|^{p+n} \\
& >r^{p}\left(1-r \sum_{n=1}^{\infty}\left|a_{p+n}\right|\right) \\
& >r^{p}-\frac{(B-A)(p-\alpha)}{1+B+(B-A)(p-\alpha)} r^{p+1} .
\end{aligned}
$$

COROLLARY 3. If $f(z)=z^{p}-\sum_{n=1}^{\infty}\left|a_{n+p}\right| z^{n+p} \in C_{p}(A, B, \alpha)$, then

$$
\begin{aligned}
& r^{p}-\frac{(B-A)(p-\alpha)(p+1)}{p[1+B+(B-A)(p-\alpha)]} r^{p+1}<|f(z)|< \\
& r^{p}+\frac{(B-A)(p-\alpha)(p+1)}{p[1+B+(B-A)(p-\alpha)]} r^{p+1}(|z|=r),
\end{aligned}
$$

with equality for

$$
f(z)=z^{p}-\frac{(B-A)(p-\alpha)(p+1)}{p[1+B+(B-A)(p-\alpha)]} z^{p+1}(z= \pm r) .
$$

THEOREM 4. The disc $|z|<1$ is mapped onto a domain that contains the disc

$|w|<\frac{1+B}{1+B+(B-A)(p-\alpha)}$ by any $f \in T_{p}^{*}(A, B, \alpha)$, and onto a domain that contains the disc $|w|<\frac{p+[p B+(A-B)(p-\alpha)]}{p \cdot[1+B+(B-A)(p-\alpha)]}$ by any $f \in C_{p}(A, B, \alpha)$.

The theorem is sharp, with extremal functions

$$
\begin{aligned}
& z^{p}-\frac{(B-A)(p-\alpha)}{1+B+(B-A)(p-\alpha)} z^{p+1} \varepsilon T_{p}^{*}(A, B, \alpha) \text { and } \\
& z^{p}-\frac{(B-A)(p-\alpha)(p+1)}{p[1+B+(B-A)(p-\alpha)]} z^{p+1} \varepsilon C_{p}(A, B, \alpha) .
\end{aligned}
$$

PROOF. The results follow upon letting $r+1$ in Theorem 3 and Corollary 3. THEOREM 5. If $\mathrm{f} \varepsilon \mathrm{T}_{\mathrm{p}}{ }^{*}(\mathrm{~A}, \mathrm{~B}, \alpha)$, then

$$
\operatorname{pr}^{p-1}-\frac{(p+1)(B-A)(p-\alpha)}{1+B+(B-A)(p-\alpha)} r^{p}<\left|f^{\prime}(z)\right|<
$$




$$
\mathrm{pr}^{p-1}+\frac{(p+1)(B-A)(p-\alpha)}{1+B+(B-A)(p-\alpha)} r^{p}(|z|=r) .
$$

Equality holds for

$$
f(z)=z^{p}-\frac{(B-A)(p-\alpha)}{1+B+(B-A)(p-\alpha)} z^{p+1}(z= \pm r)
$$

PROOF. We have

$$
\begin{aligned}
& \left|f^{\prime}(z)<p r^{p-1}+\sum_{n=1}^{\infty}(p+n)\right| a_{p+n} \mid r^{p+n-1} \\
& <p r^{p-1}+r^{p} \sum_{n=1}^{\infty}(p+n)\left|a_{p+n}\right| \\
& =r^{p-1}\left[p+r \sum_{n=1}^{\infty}(p+n)\left|a_{p+n}\right|\right] .
\end{aligned}
$$

In view of Theorem 1 ,

$$
\begin{aligned}
& \sum_{n=1}^{\infty}(1+B)\left[n+p-\frac{p(1+B)+(A-B)(p-\alpha)}{1+B}\right]\left|a_{n+p}\right| \\
& <(B-A)(p-\alpha)
\end{aligned}
$$

or

$$
\begin{aligned}
& \sum_{n=1}^{\infty}(1+B)(n+p)\left|a_{n+p}\right|<(B-A)(p-\alpha)+ \\
& {[p(1+B)+(A-B)(p-\alpha)] \sum_{n=1}^{\infty}\left|a_{n+p}\right|}
\end{aligned}
$$

(4.4) with the help of (4.2) implies that

$$
\sum_{n=1}^{\infty}(n+p)\left|a_{n+p}\right|<\frac{(p+1)(B-A)(p-\alpha)}{1+B+(B-A)(p-\alpha)}
$$

A substitution of (4.5) into (4.3) yields the right-hand inequality.

On the other-hand

$$
\begin{aligned}
& \left|f^{\prime}(z)\right|>r^{p-1}\left[p-r \sum_{n=1}^{\infty}(p+n)\left|a_{p+n}\right|\right] \\
& >p r^{p-1}-\frac{(p+1)(B-A)(p-\alpha)}{1+B+(B-A)(p-\alpha)} r^{p} .
\end{aligned}
$$

This completes the proof. 

then

COROLlaRY 4. If $f(z)=z^{p}-\sum_{n=1}^{\infty}\left|a_{n+p}\right| z^{n+p} \varepsilon C_{p}(A, B, \alpha)$,

$$
\begin{aligned}
& p r^{p-1}-\frac{(B-A)(p-\alpha)(p+1)^{2}}{p[1+B+(B-A)(p-\alpha)]} r^{p}<\left|f^{\prime}(z)\right|< \\
& p r^{p-1}+\frac{(B-A)(p-\alpha)(p+1)^{2}}{p[1+B+(B-A)(p-\alpha)]} r^{p}(|z|=r) .
\end{aligned}
$$

Equality holds for $f(z)=z^{p}-\frac{(B-A)(p-\alpha)(p+1)}{p[1+B+(B-A)(p-\alpha)]} z^{p+1}(z= \pm r)$.

5. RADIUS OF CONVEXITY FOR THE ClASS $T_{p}^{*}(A, B, \alpha)$.

THEOREM 6. If $f(z) \varepsilon T_{p}^{*}(A, B, \alpha)$, then $f(z)$ is p-valently convex in the disc

$$
|z|<R_{p}=\inf _{n}\left[\frac{(1+B) n+(B-A)(p-\alpha)}{(B-A)(p-\alpha)}\left(\frac{p}{n+p}\right)^{2}\right]^{\frac{1}{n}} \quad(n=1,2, \ldots) .
$$

The result is sharp, with the extrenal function

$$
f(z)=z^{p}-\frac{(B-A)(P-\alpha)}{(1+B) n+(B-A)(p-\alpha)} z^{p+n}
$$

PROOF. It is sufficient to show that $\mid\left(1+\frac{z f^{\prime \prime}(z)}{f^{\prime}(z)}-p \mid<p\right.$ for $|z|<R_{p}$.

We have

$$
\begin{aligned}
\left|\left(1+\frac{z f^{\prime \prime}(z)}{f^{\prime}(z)}\right)-p\right| & =\frac{-\sum_{n=1}^{\infty} n(n+p)\left|a_{n+p}\right| z^{n}}{p-\left.\sum_{n=1}^{\infty}(n+p)\left|a_{n+p}\right| z\right|^{n}} \\
& <\frac{\sum_{n}^{\infty} n(n+p)\left|a_{n+p}\right||z|^{n}}{p-\sum_{n=1}^{\infty}(n+p)\left|a_{n+p}\right||z|^{n}} .
\end{aligned}
$$

Thus

$$
\begin{aligned}
& \left|\left(1+\frac{z f^{\prime \prime}(z)}{f^{\prime}(z)}\right)-p\right|<p \text { if } \\
& \sum_{n=1}^{\infty}(n+p)^{2}\left|a_{n+p}\right||z|^{n}<p^{2}
\end{aligned}
$$

or

$$
\sum_{n=1}^{\infty}\left(\frac{n+p}{p}\right)^{2}\left|a_{n+p}\right||z|^{n}<1
$$

Ac cording to Theorem 1, $\sum_{n=1}^{\infty} \frac{(1+B) n+(B-A)(p-\alpha)}{(B-A)(p-\alpha)}\left|a_{n+p}\right|<1$. 
Hence (5.2) will be true if

$$
\left(\frac{n+p}{p}\right)^{2}|z|^{n}<\frac{(1+B) n+(B-A)(p-\alpha)}{(B-A)(p-\alpha)}
$$

or if

$$
|z|<\left[\frac{(1+B) n+(B-A)(p-\alpha)}{(B-A)(p-\alpha)} \cdot\left(\frac{p}{n+p}\right)^{2}\right]^{\frac{1}{n}} \quad(n=1,2, \ldots)
$$

The theorem follows easily from (5.3).

6. CLOSURE THEOREMS.

In this section we shall prove that the classes $T_{p}^{*}(A, B, \alpha)$ and $C_{p}(A, B, \alpha)$ are closed under convex linear combinations.

THEOREM 7. If $f(z)=z^{p}-\sum_{n=1}^{\infty}\left|a_{n+p}\right| z^{n+p}$ and $g(z)=z^{p}-\sum_{n=1}^{\infty}\left|b_{n+p}\right| z^{n+p}$ are in $T_{p}^{*}(A, B, \alpha)$, then $h(z)=z^{p}-\frac{1}{2} \sum_{n=1}^{\infty}\left|a_{n+p}+b_{n+p}\right| z^{n+p}$ is also in $T_{p}^{*}(A, B, \alpha)$.

PROOF. Since $f(z)$ and $g(z)$ are in $T_{p}^{*}(A, B, \alpha)$, we have

$$
\sum_{n=1}^{\infty}[(1+B) n+(B-A)(p-\alpha)]\left|a_{n+p}\right|<(B-A)(p-\alpha)
$$

and

$$
\sum_{n=1}^{\infty}[(1+B) n+(B-A)(p-\alpha)]\left|b_{n+p}\right|<(B-A)(p-\alpha) .
$$

From (6.1) and (6.2) we get

$$
1 / 2 \sum_{n=1}^{\infty}[(1+B) n+(B-A)(p-\alpha)]\left|a_{n+p}+b_{n+p}\right|<(B-A)(p-\alpha)
$$

which implies that $h(z) \in T_{p}^{*}(A, B, \alpha)$.

The following theorem can be proven similarly.

THEOREM 8. $f(z)=z^{p}-\sum_{n}^{\infty}\left|a_{n+p}\right| z^{n+p}$ and $g(z)=z^{p}-\sum_{n=1}^{\infty}\left|b_{n+p}\right| z^{n+p}$ are in $C_{p}(A, B, \alpha)$, then $h(z)=z^{p} 1 / 2 \sum_{n=1}^{\infty}\left|a_{n+p}+b_{n+p}\right| z^{n+p}$ is also in $C_{p}(A \cdot B \cdot \alpha)$.

THEOREM 9. Let $f_{p}(z)=z^{p}, f_{n+p}(z)=z^{p}-\frac{(B-A)(p-\alpha)}{(1+B) n+(B-A)(p-\alpha)} z^{n+p}(n=1,2,3, \ldots)$. Then $f \varepsilon T_{p}^{*}(A, B, \alpha)$ if and only if it can be expressed in the form

$$
f(z)=\sum_{n=0}^{\infty} \lambda_{n+p} f_{n+p}(z) \text { where } \lambda_{n+p}>0 \text { and } \sum_{n=0}^{\infty} \lambda_{n+p}=1 \text {. }
$$

PROOF. Suppose $f(z)=\sum_{n=0}^{\infty} \lambda_{n+p} f_{n+p}(z)=z^{p} \sum_{n=0}^{\infty} \frac{(B-A)(p-\alpha)}{(1+B) n+(B-A)(p-\alpha)} \lambda_{n+p} z^{n+p}$. 
Then

$$
\begin{aligned}
& \sum_{n=1}^{\infty}\left[\lambda_{n+p} \frac{(1+B) n+(B-A)(p-\alpha)}{(B-A)(p-\alpha)} \cdot\left(\frac{(B-A)(p-\alpha)}{(1+B) n+(B-A)(p-\alpha)}\right)\right] \\
& =\sum_{n=1}^{\infty} \lambda_{n+p} \leqslant 1-\lambda_{p} \leqslant 1 .
\end{aligned}
$$

So by Theorem 1, $f(z) \varepsilon T_{p}^{*}(A, B, \alpha)$.

$$
\text { Conversely suppose } f(z) \varepsilon T_{p}^{*}(A, B, \alpha) \text {. Then }
$$

$$
\left|a_{n+p}\right|<\frac{(B-A)(p-\alpha)}{(1+B) n+(B-A)(p-\alpha)}
$$

Se tting $\lambda_{n+p}=\frac{(1+B) n+(B-A)(p-\alpha)}{(B-A)(p-\alpha)}\left|a_{n+p}\right|(n=1,2, \ldots)$,

and

$$
\lambda_{p}=1-\sum_{n=1}^{\infty} \lambda_{n+p},
$$

we have

$$
f(z)=\sum_{n=0}^{\infty} \lambda_{n+p} f_{n+p}(z)
$$

This completes the proof of the theorem.

REMARKS. (1) Putting $\alpha=0$ in the above theorems we get the results obtained by R.M. Goel and N.S. Sohi [1].

(2) Putting $p=1$ and taking $A=-B, B=B$, where $0<\beta<1$, in the above theorems we get the results obtained by Gupta and Jain [2].

(3) Putting $p=1$ and taking $A=-1, B=1$ in the above theorems we get the results obtained by Silverman [3].

ACKNOWLEDGMENT. In conclusion, I would like to thank Professor Dr. H. Silverman for reading the manuscript and for helpful suggestions.

\section{REFERENCES}

1. GOEL, R.M. and SOHI, N.S. Multivalent functions with negative coefficients, Indian J. Pure appl. Math., 12(7) (1981), 844-853.

2. GUPTA, V.P. and JAIN, P.K. Certain classes of univalent functions with negative coefficients, Bul1. Austr. Math. Soc., 14 (1976), 409-416. 
3. SILVE RMAN, H. Unjvalent functions with negative coefficients, Proc. Amer. Math. Soc. 51 (1975), 109-116.

4. SILVERMAN, H. and SILVIA, E.M. Convex families of starlike functions, Houston J. Math., 4, (1978), 263-268.

5. SILVERMAN, H. and SILVIA, E.M. Pre-Starlike functions with negative coefficients Int. J. Math. Sci., 2 (1979), 427-439. 


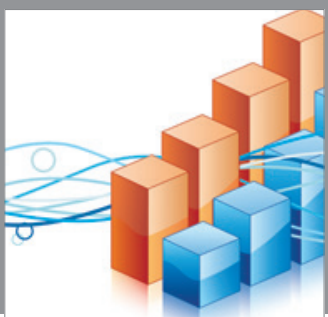

Advances in

Operations Research

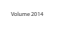

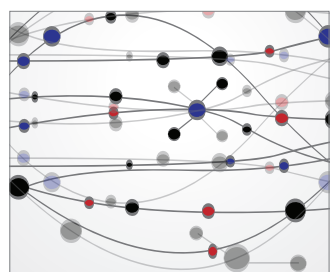

\section{The Scientific} World Journal
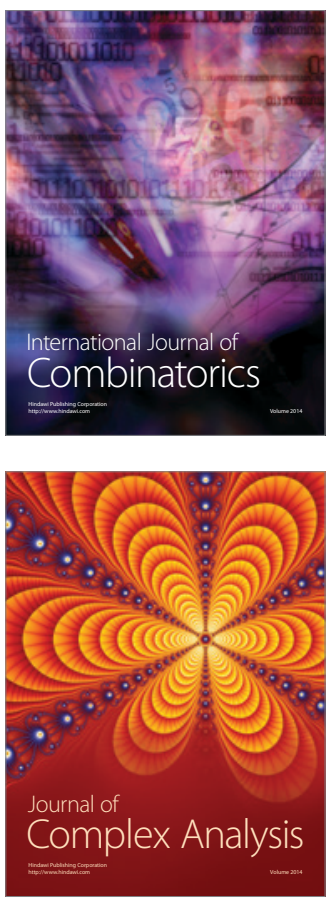

International Journal of

Mathematics and

Mathematical

Sciences
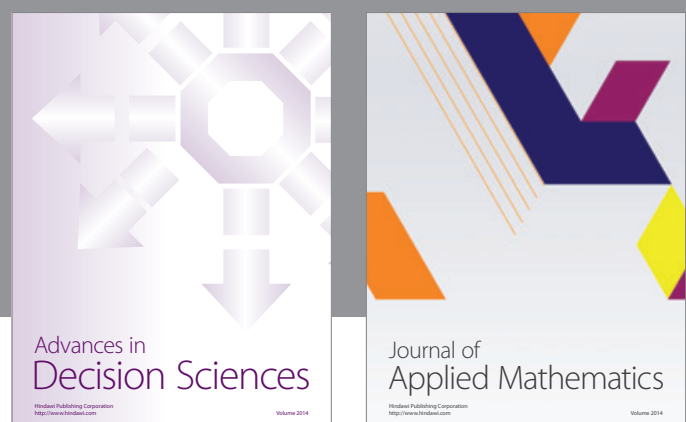

Journal of

Applied Mathematics
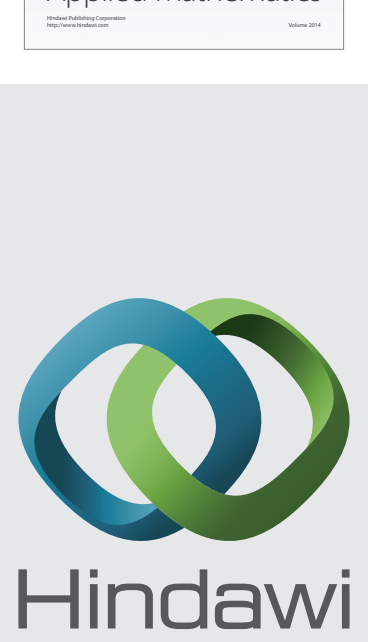

Submit your manuscripts at http://www.hindawi.com
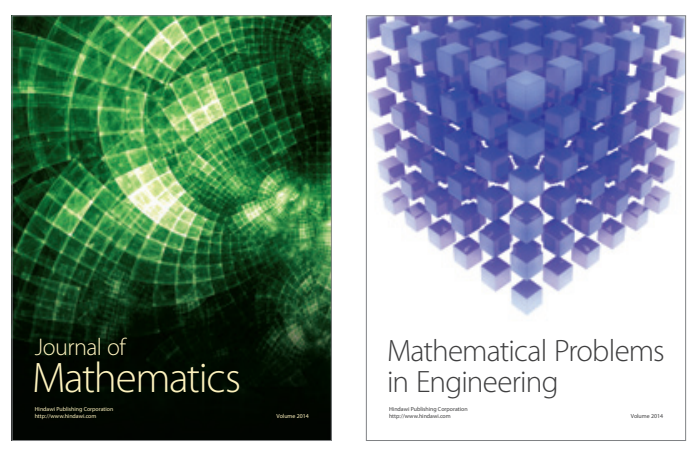

Mathematical Problems in Engineering
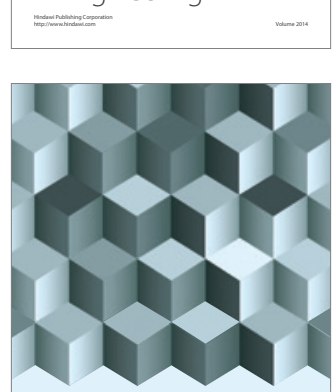

Journal of

Function Spaces
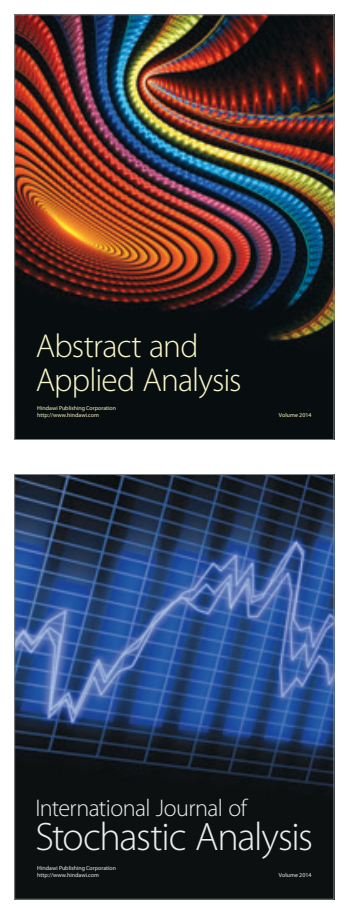

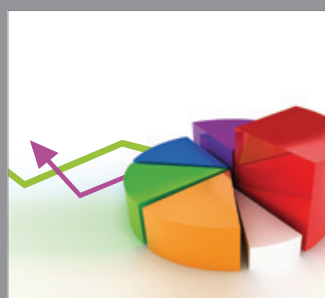

ournal of

Probability and Statistics

Promensencen
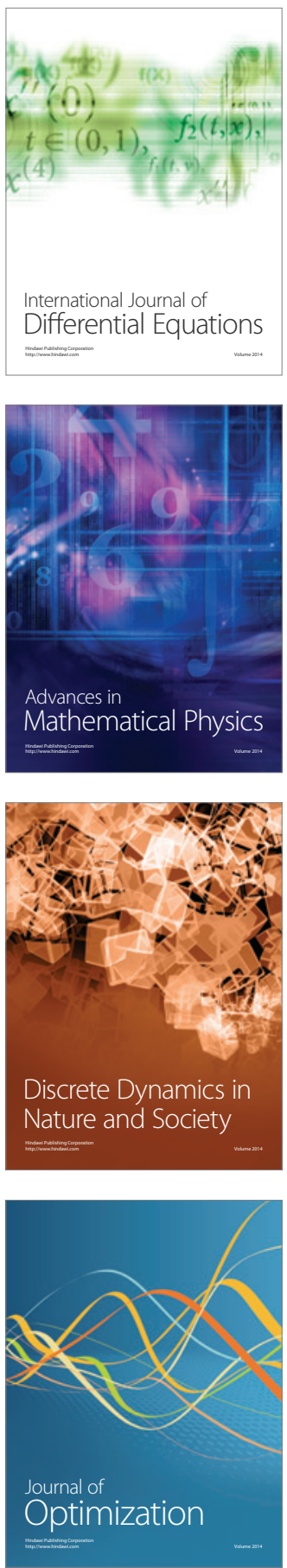